\section{Angst}

Das wichtigste Akutmittel ist zweifellos Anxiovita ${ }^{\circledR}$ (in der Schweiz als Kava Kava comp. erhältlich). Als spezifisch wirkendes homöopathisches Komplexmittel für Angstzustände hat es sich bei Panikzuständen, Prüfungsangst, sozialer Phobie und anderen Formen von Angst bewährt. Üblicherweise gibt man zweimal täglich 12 Tropfen bzw. bei akuten Beschwerden zusätzlich alle 30 min 5 Tropfen bzw. Globuli. Nach der Erfahrung an Hunderten von Patienten wirkt es meist zuverlässig und schnell, sodass es beispielsweise bei Angstkranken als vorbeugendes Notfallmittel eingesetzt werden kann. Das gilt auch bei situativer Angst etwa kurz vor einer Prüfung, wo es ebenfalls vorbeugend wirkt.

Die jahrelange Erfahrung hat gezeigt, dass Anxiovita spezifisch bei Angst wirkt und hierbei auch diagnostisch eingesetzt werden kann. Wenn man es in der allgemeinmedizinischen Praxis regelmässig an Patienten energetisch testet, etwa durch den kinesiologischen Muskeltest, stellt man überraschend fest, dass es bei mehr Patienten anspricht, als man von der Anamnese her erwarten würde. Wenn man genauer nachfragt, handelt es sich gleichwohl um Angstkranke, die durch unterschwellige

\title{
Die Akutmittel der Psychosomatischen Energetik
}

In der Psychosomatischen Energetik (PSE) werden vier homöopathische Komplexe als «Akutmittel» bezeichnet, weil man sie bei akuten Beschwerden verwendet. Die Akutmittel stehen von der Darreichungsform als apothekenpflichtige Spezialitäten sowohl als Globuli sowie auch als Tropfen in Flaschen à $50 \mathrm{ml}$ zur Verfügung und werden von der Rubimed ${ }^{\circledR}$ AG (Schweiz) bzw. Rubimed ${ }^{\circledR}$ B.V. (EU-Raum) vertrieben. Wie man diese Spezialitäten nutzbringend in der täglichen Praxis einsetzt, und das auch ohne die Anwendung der PSE, möchte ich im folgenden Beitrag erklären.

Befürchtungen etwa um nahe Angehörige oder um die Sicherheit des Arbeitsplatzes in inneren Stress versetzt worden sind, was sie unruhig und angespannt macht. Das führt dann oft irgendwann zu angstuntypischen Symptomen. Solche Patienten berichten von Erschöpfung, Anspannung, Schmerz, Unruhe usw., wobei den geschilderten Beschwerden primär eine Angst zugrunde liegt, was das positive Ansprechen auf die Testampulle Anxiovita sowie später das positive Ansprechen auf eine angstlösende Therapie bestätigt.

Nicht selten erlebt man auch bei diversen Suchtformen, dass die AngstAmpulle Anxiovita anspricht. Dann dient beispielsweise ein krankhafter Alkoholkonsum dazu, die Angst in Form einer wenig effizienten Eigentherapie zu bewältigen. Verständlicherweise sollte man bei allen Fällen von Angst an der seelischen Ursache ansetzen, etwa durch Gabe von Anxiovita, durch angstlösende Gespräche sowie durch das Auflösen seelischer Konflikte durch die PSE.

\section{Nervöse Anspannung und Übererregung}

Bei bestimmten akuten Krankheiten des Nervensystems, etwa bei einer Multiplen Sklerose, Polyneuropathie oder Trigeminusneuralgie, sowie bei chronischen Schmerzzuständen wie einer Migräne, Neuralgie usw. spricht Neurovita ${ }^{\circledR}$ im energetischen Medikamententest an (in der Schweiz als Melissa comp. erhältlich). Es bewährt sich therapeutisch zur allgemeinen Beruhigung und auch zur Regeneration.

\section{Autonome Fehlregulation}

Das autonome Nervensystem (ANS) regelt einer Klimaanlage vergleichbar zentrale Körperfunktionen wie Schlaf, Hauttemperatur, Verdauung, Stoffwechsel und Kreislauffunktionen wie Blutdruck und Herzschlag. Menschen mit vegetativen Störungen klagen über so unterschiedliche Beschwerden wie kalte Hände, Schwitzen, eine beschleunigte oder verlangsamte Verdauungstätigkeit und zahlreiche andere Befindlichkeitsstörungen. Da das vegetative Nervensystem für unser Wohlbefinden verantwortlich zeichnet, fühlen sich Betroffene oft permanent unwohl.

Wichtig ist es, vegetative Überund Unterfunktionen voneinander zu unterscheiden, weil sich daraus unterschiedliche Therapiestrategien ergeben. Mit Simvita ${ }^{\circledR}$ werden sympathikotone Störungen aufgedeckt, die mit einer «überhitzten Klimaanlage»

\section{KARGER}

Fax +497614520714 Information@Karger.de www.karger.com
(๑) 2012 S. Karger GmbH, Freiburg

Accessible online at: www.karger.com/szg
Dr. med. Reimar Banis

Arzt für Allgemeinmedizin/Naturheilverfahren

c/o Internationale Gesellschaft für Psychosomatische Energetik (IGPSE)

Dörflistrasse 4, 6056 Kägiswil, Schweiz

www.igpse.ch 
verglichen werden können (mit Symptomen wie Schwitzen, Tachykardie, Diarrhö, Unruhe, Gewichtsabnahme). Im Unterschied dazu werden mit Paravita ${ }^{\circledR}$ parasympathikotone Dysfunktionen angesprochen, was einer $\mathrm{zu}$ stark kühlenden Klimaanlage gleicht (mit Symptomen einer trockenen Haut, Frieren, Bradykardie, Kollapsneigung, Obstipation und Gewichtszunahme). Wegen der funktionellen Gegenregulation des vegetativen Nervensystems, das auf unterschiedlicher Ebene intakt und auf anderen gestört sein kann, sollte man wissen, dass nicht alle genannten Symptome auftreten, sodass in der Praxis zusätzliche diagnostische Wegweiser nötig sind.

Mithilfe des kinesiologischen Ansprechens der Testampullen von Simvita bzw. Paravita (im Basistestkasten - mit allen Akutmitteln - der Firma Rubimed enthalten; Rubimed AG, Grossmatt 3, 6052 Hergiswil, Schweiz; www.rubimed.com) können die entsprechenden Fehlfunktionen erkannt und später damit therapiert werden. Eine naturheilkundliche Therapie des ANS ist allein schon deshalb sinnvoll, da es in der Schulmedizin weder eine objektive Diagnose noch eine Therapie der autonomen Fehlfunktion gibt. Daher werden viele derartige Beschwerden in der Alltagspraxis entweder nicht richtig diagnostiziert, als psychogen abgetan und oft aus der Sicht der Betroffenen unbefriedigend therapiert. Wegen des grossen subjektiven Leidensdrucks ist ein schneller Therapieerfolg wünschenswert, weshalb Simvita bzw. Paravita in einer Dosis von 2-3 $\times 12$ Tropfen täglich verabreicht oft eine rasche Linderung bringen (in der Schweiz als Adrenalin bzw. Acetylcholin comp. erhältlich).

\section{Schlussfolgerung}

Die vier Akutmittel der PSE können sowohl rein symptomatisch aufgrund bestimmter Beschwerden als auch aufgrund energetischer Testergebnisse (unter anderem kinesiologischer Medikamententest) eingesetzt werden. Sie haben sich in der täglichen, naturheilkundlich ausgerichteten Praxis als rasch wirksame Heilmittel bewährt. Mit den vegetativ wirkenden Komplexen Simvita und Paravita können oft auch akute Phasen eines grippalen Infekts kupiert bzw. verkürzt werden, indem es dadurch schneller zu einer autonomen Umschaltung und Harmonisierung kommt. Anxiovita verhilft dazu, sonst unerkannte chronische Angsterkrankungen aufzudecken und kausal zu therapieren.

\section{Literatur}

Banis R: Psychosomatische Energetik. Lehrbuch für Therapeuten, ed 2. Kirchzarten, VAK, 2005. Hartmann E: Über Konstitutionen, Yin Yang und Reaktionstypen. Waldbrunn, Eigenverlag im Forschungskreis für Geobiologie Waldbrunn, 1986.

Thews V: Vegetative Physiologie. Heidelberg, Springer, 2001. 